\title{
In vitro release of arachidonic acid metabolites, glutathione peroxidase, and oxygen-free radicals from platelets of asthmatic patients with and without aspirin intolerance
}

Vicente Plaza, Jordi Prat, Joan Rosellò, Eugeni Ballester, Isabel Ramis, Joaquim Mullol, Emilio Gelpí, Josep L L Vives-Corrons, César Picado

\begin{abstract}
Background - An abnormal platelet release of oxygen-free radicals has been described in acetylsalicylic acid (aspirin)induced asthma, a finding which might suggest the existence of an intrinsic, specific platelet abnormality of arachidonic acid metabolism in these patients. The objective of this study was to evaluate platelet arachidonic acid metabolism in asthmatic patients with or without intolerance to aspirin.

Methods - Thirty subjects distributed into three groups were studied: group 1, 10 healthy subjects; group 2, 10 asthmatic patients with aspirin tolerance; and group 3, 10 aspirin-intolerant asthmatics. Platelets were isolated from blood, preincubated with ${ }^{3} \mathrm{H}$-arachidonic acid for 30 minutes and then incubated for 10 minutes with platelet activating factor (PAF) and aspirin. Cyclo-oxygenase (thromboxane, PGE, PGF $_{2 \alpha}$, and HHT) and lipoxygenase (12-HETE) arachidonic acid metabolites were measured by high pressure liquid chromatography. Release of oxygen free radicals after incubation with PAF and aspirin was measured by chemiluminescence. Platelet levels of glutathione peroxidase (GSH-Px) were also measured using spectrophotometry.

Results - Platelets from aspirin-intolerant asthmatic patients produced higher quantities of arachidonic acid metabolites than the control group at baseline conditions. This increase was significant only for lipoxygenase products. No differences were found amongst the three groups in the response of arachidonic acid metabolism to PAF and aspirin. Incubation with aspirin but not with PAF caused an increase in oxygen-free radical production in aspirin-intolerant patients whereas in aspirin-tolerant patients PAF, rather than aspirin, was the more potent stimulus for oxygen-free radical production. No differences in GSH-Px levels were found amongst the three groups.
\end{abstract}

Conclusions - These results suggest that the platelet lipoxygenase pathway is ac- tivated in aspirin-intolerant patients and that the production of oxygen-free radicals may differentiate aspirin-tolerant from as $-\vec{\theta}$ pirin-intolerant asthmatic subjects. Our study, however, does not support the hypothesis that an increase in lipoxygenase products may be responsible for oxygenfree radical production. Moreover, a lowered platelet GSH-Px activity does not seem to be involved in this phenomenon. (Thorax 1995;50:490-496)

Keywords: bronchial asthma, aspirin-sensitive asthma, oxygen-free radicals, arachidonic acid metabolites, glutathione peroxidase.

In some asthmatic patients acetylsalicylic acid (aspirin) and other non steroidal anti-in- $O$ flammatory drugs (NSAIDs) precipitate asthma attacks. ${ }^{1-4}$ Although during the last two decades numerous studies have been carried $\frac{D}{O}$ out to investigate this phenomenon, the mechanism(s) responsible remains unknown. 0 Szczeklik et $a l^{5}$ showed that all drugs that share N the common property of inhibiting the cyclo- N oxygenase pathway can provoke asthma 0 attacks. This finding has suggested that, in aspirin-induced asthma, blockade of the cyclo- $\frac{}{\square}$ oxygenase enzyme might divert arachidonic? acid metabolism from the cyclo to the lip- $\frac{T}{7}$ oxygenase pathway, leading to both an $\frac{\vec{D}}{\mathbb{D}}$ excessive production of bronchoconstrictor leukotrienes as well as a lack of bronchodilator $\stackrel{\mathbb{Q}}{2}$ $\mathrm{PGE}_{2}{ }^{13}$ It has also been proposed that aspirin intolerance is the consequence of viral in- 8 fection, ${ }^{6}$ but this hypothesis has yet to be substantiated by any experimental evidence. A

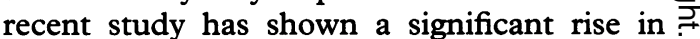
urinary $\mathrm{LTE}_{4}$ levels after aspirin ingestion in aspirin-sensitive patients. ${ }^{7}$ Picado et $a l^{8}$ have also reported that intranasal challenge with lysine-aspirin is associated with an increased concentration of peptide leukotrienes in nasal lavage in aspirin-sensitive subjects. The demonstration of increased leukotriene release in aspirin-induced asthma suggests that peptide 
leukotrienes have a role in the pathogenesis of aspirin sensitivity. The cellular source for the augmented leukotriene production is still unknown.

Although the role of platelets in aspirininduced asthma is a matter of debate, some recent findings suggest that these cells might be involved in this peculiar clinical syndrome. Ameisen $e t a l^{910}$ have described an abnormal platelet release of oxygen-free radicals from aspirin-sensitive asthmatics exposed to aspirin in vitro - a finding that might suggest the existence of an intrinsic, specific platelet abnormality of arachidonic acid metabolism in these patients. Recent studies have also shown lowered glutathione peroxidase (GSH-Px) activity in platelets of aspirin-sensitive asthmatics $^{112}$ and, since GSH-Px plays an important part in the protection against oxidative damage, ${ }^{13-15}$ it has been suggested that aspirinrelated asthma attacks are due in part to a deficit in this scavenger. ${ }^{112}$ On the other hand, although no consistent abnormality in arachidonic acid metabolism has ever been shown in aspirin-sensitive patients, it seems reasonable to suspect that the abnormal response of a patient's platelets to NSAIDs is due to a change in the balance of arachidonic acid metabolism. If this hypothesis was true we might expect a different pattern of release of arachidonic acid metabolites from the platelets of aspirin-sensitive patients compared with both aspirin-insensitive asthmatics and healthy subjects.

Because release of oxygen-free radicals can occur after activation of both the cyclo and lipoxygenase pathways, we reasoned that aspirin must increase the production of oxygenfree radicals in aspirin-intolerant asthmatic subjects by increasing the activity of either one or both pathways. In order to assess this potential aspirin action we compared the effects of aspirin with platelet activating factor (PAF), a well known potent platelet activator.

It is interesting to note that the three postulated intrinsic platelet abnormalities (excessive production of oxygen-free radicals, lowered GSH-Px activity, and an increased production of lipoxygenase metabolites) have never been evaluated in a study designed to assess their interrelation in the pathogenesis of aspirin-induced idiosyncratic reactions. The aim of this study was to evaluate these abnormalities and their possible relationship in the origin of aspirin-induced asthma.

Mean (SD) characteristics of subjects

\begin{tabular}{llll}
\hline & Group 1 & Group 2 & Group 3 \\
\hline $\mathrm{n}$ & 10 & 10 & 10 \\
Age (years) & $39 \cdot 4(7)$ & $38 \cdot 6(16)$ & $40 \cdot 2(12)$ \\
\% Male/female & $45 / 55$ & $45 / 55$ & $45 / 55$ \\
Smoker or ex-smoker (\%) & 25 & 70 & 10 \\
Rhinitis (\%) & 13 & 90 & 100 \\
Years of rhinitis & - & $13(13)$ & $18 \cdot 2(16)$ \\
Nasal polyps (\%) & - & 10 & $73 *$ \\
Years of asthma & - & $11 \cdot 1(5 \cdot 4)$ & $11 \cdot 2(10 \cdot 1)$ \\
Inhaled steroid treatment (\%) & - & 36 & 64 \\
FEV (\%predicted) & - & $77(35)$ & $83(13)$ \\
Prick test (+)(\%) & - & 70 & 30 \\
Eosinophils in blood & - & $228(201)$ & $363(355)$ \\
Score severity of the disease & - & $3 \cdot 4(1 \cdot 9)$ & $4 \cdot 4(2 \cdot 1)$ \\
\hline
\end{tabular}

Severity of the disease was assessed taking into account clinical symptoms (score 1-3), degree of bronchial obstruction $(1-4)$ and the amount of treatment needed to control symptoms (1-3). * $p<0.005$ ( $\chi^{2}$ test $)$.

\section{Methods}

\section{SUBJECTS}

Ten healthy subjects (group 1), 10 aspirintolerant asthmatic patients (group 2), and 10 aspirin-intolerant asthmatic patients (group 3) were included in the study. Characteristics of the three groups are depicted in the table. Asthma was diagnosed when there was a history of shortness of breath, wheezing, and evidence of reversible airways obstruction. Diagnosis of aspirin intolerance was made on the basis of a clear history of two or more acute asthmatic attacks precipitated by aspirin or other NSAIDs. Aspirin intolerance was confirmed by nasal challenge with aspirin according to a previously described method. ${ }^{8}$ Only patients with a predominant asthmatic response were included (none had had isolated urticarial/anaphylactic reactions without asthmatic exacerbation). Aspirin-tolerant asthmatics denied any aspirin-induced reaction. The healthy volunteers were recruited for the study from the staff of our institution. The criteria for inclusion were current good health and absence of any treatment at the time of recruitment. All subjects gave informed and signed consent to participate in the study, which was approved by the ethical committee of our institution.

The three groups were similar in age and sex distribution. As expected, the proportion of aspirin-intolerant patients suffering from nasal polyps was higher than in the aspirin-tolerant group. Severity of disease was assessed according to a score taking into account clinical symptoms (score 1-3), severity of bronchial obstruction measured by forced spirometry (score 1-4), and the amount of treatment needed to control symptoms (score 1-3). There were no differences between tolerant and intolerant patients with respect to either clinical score or bronchial obstruction. A higher proportion of aspirin-intolerant patients received treatment with inhaled steroids, but the difference was not significant. None of them, however, were on oral steroid treatment.

\section{PLATELET ISOLATION}

Between 08.30 and 09.30 hours a $30 \mathrm{ml}$ specimen of blood was drawn from the antecubital vein of each of the 30 subjects. Platelet-rich plasma was obtained by a method previously described by Levine and Fedorko ${ }^{16}$ and modified by Rao et al. ${ }^{17}$ Briefly, whole blood was centrifuged at $225 \mathrm{~g}$ for 15 minutes at $37^{\circ} \mathrm{C}$. In order to discard anomalous platelets and previous ingestion of any antiaggregant substance, aggregation studies were performed by adding arachidonic acid to $450 \mu$ l platelet-rich plasma according to Born's method ${ }^{18}$ (Aggrecoder PA-3210 Hitachi). Platelets from the platelet-rich plasma were washed twice with equal volumes of citrate-citric acid-dextrose $(93 \mathrm{mmol} / 1$ sodium citrate, $70 \mathrm{mmol} / 1 \mathrm{citric}$ acid, and $140 \mathrm{mmol} / 1$ dextrose), $\mathrm{pH} 6.5$, containing $5 \mathrm{mmol} / 1$ adenosine and $3 \mathrm{mmol} / 1$ theophylline and centrifuged at $750 \mathrm{~g}$ for 15 minutes at $37^{\circ} \mathrm{C} .{ }^{19}$ The final pellet was resuspended in Hank's balanced salt solution to a final platelet count of $300 \times 10^{6}$ platelets $/ \mathrm{ml}$. 


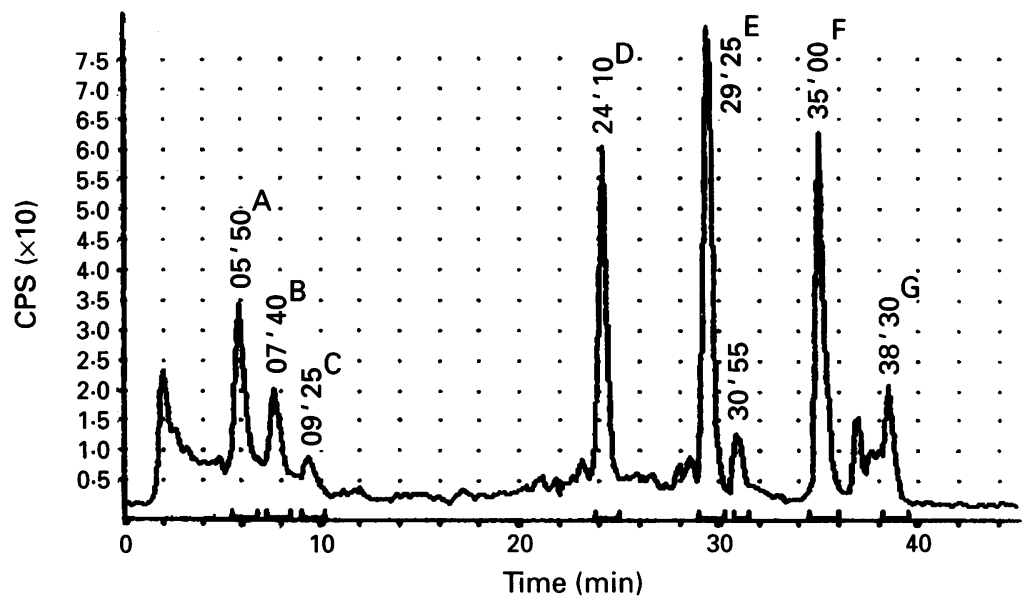

Figure 1 HPLC profile of platelet prostanoids from an aspirin-intolerant patient after stimulation with platelet activating factor (PAF). Quantitation of ${ }^{3} \mathrm{H}$-labelled metabolites was performed using a radiochromatographic system (see methods for more details). Peaks: $A=$ thromboxane; $B=P G F_{22} ; C=P G E_{2} ; D=H H T ; E=12-H E T E ; F=$ metabolite $X$; and $G=$ arachidonic acid. Metabolite $X$ corresponds to an unidentified metabolite. Preliminary studies suggest that this compound is a lipoxygenase product. CPS $=$ counts per second. pumps (Applied Biosystems, Ramsey, New Jersey, USA) and products were monitored with a radioactivity detector (Ramona, Raytest, Straubenhardt, Germany). The metabolites were measured using a radiochromatographic 걱 system from Nuclear Interface (Münster, Ger- 응 many). Formic acid $40 \mathrm{mM}$ titrated to $\mathrm{pH} 3 \cdot 15 \stackrel{\text {. }}{\text {. }}$ with triethylamine/acetonitrile was employed $\vec{F}$ as a mobile phase. The elution conditions involved an isocratic initial elution for 14 minutes $\frac{0}{5}$ (33\% acetonitrile), a linearly increasing aceto- $\frac{\bar{c}}{\bar{D}}$ nitrile gradient (33-60\%) for eight minutes, $\mathbb{\Phi}$ an isocratic elution for nine minutes $\left(60 \% \frac{0}{0}\right.$ acetonitrile), a linearly increasing acetonitrile gradient $(60-100 \%)$, and an isocratic elution. for five minutes (100\% acetonitrile). The flow $\overrightarrow{\vec{\omega}}$ rate was $1.5 \mathrm{ml} / \mathrm{min}$. Reversed phase chromatography was performed on a Spherisorb $\overrightarrow{\vec{x}}$ ODS-2 (250 $4.6 \mathrm{~mm}, 10 \mu \mathrm{m})$ column (Phase 용 Separations Ltd, Deeside, UK). These con- or ditions allowed a total separation of pro- $\vec{c}$ stanoids, HHT, 12-HETE, and arachidonic acid. Figure 1 shows a chromatogram of a patient with NSAID intolerance after stimu- $\vec{z}$ lation by PAF. An unidentified peak appeared at 35 minutes retention time (metabolite $\mathrm{X}$ ). $\overrightarrow{0}$ In order to identify this compound the fraction 8 corresponding to its retention time was purified as well as the $\mathrm{TXB}_{2}, \mathrm{HHT}$, and 12-HETE fractions. Preliminary studies suggest that this compound is a lipoxygenase metabolite. arachidonic acid $(2 \mu \mathrm{Ci} /$ tube) by shaking for 30 minutes at $37^{\circ} \mathrm{C}$ as previously reported. ${ }^{20}$ Aspirin $(50 \mu \mathrm{M} / 20 \mu \mathrm{l})$ and PAF $(10 \mu \mathrm{M} / 50 \mu \mathrm{l})$ were then added to the three tubes. One tube was used as a control. $\mathrm{Ca}^{2+}$ and $\mathrm{Mg}^{2+}$ salts were added to each tube and incubation was carried out for 10 minutes before stopping the reaction by adding $2 \mathrm{ml} \mathrm{Hank's} \mathrm{solution} \mathrm{at} 4^{\circ} \mathrm{C}$. An incubation time of 10 minutes was used because the maximum production of both cyclo and lipoxygenase products after PAF stimulation occurred at this time in a preliminary study in three subjects. After centrifugation at $1000 \mathrm{~g}$ for 15 minutes at $4^{\circ} \mathrm{C}$ supernatants were stored at $-70^{\circ} \mathrm{C}$ until assay. The effects of $\mathrm{PAF}$ and aspirin on arachidonic acid metabolite synthesis were expressed as increments with respect to the values obtained from the control tube.

\section{EXTRACTION AND PURIFICATION}

Eicosanoid extraction was carried out according to methods previously described. ${ }^{21}$ Briefly, supernatants of platelet incubations were acidified to $\mathrm{pH} 4$ with $1 \mathrm{~N} \mathrm{HCl}$ and processed through $\mathrm{C} 18$ reversed phase cartridges (Baker, Phillipsburg, New Jersey, USA) which had been pretreated with both $10 \mathrm{ml}$ methanol and $10 \mathrm{ml}$ water ( $\mathrm{pH} 4$ ). After sample addition, cartridges were washed with $10 \mathrm{ml}$ water and eicosanoids were eluted with $5 \mathrm{ml}$ methanol and evaporated under helium stream. Dried residues were stored at $-70^{\circ} \mathrm{C}$ until HPLC assay.

HIGH PRESSURE LIQUID CHROMATOGRAPHY (HPLC) ANALYSIS

HPLC separation of ${ }^{3} \mathrm{H}$-labelled metabolites was performed using two Spectroflow 400

\section{CHEMILUMINESCENCE ASSAY}

Platelet chemiluminescence was measured according to the method of Ameisen et $a l^{9}$ with a few modifications. Light emission was measured in a Monolight 2001 Lumac (Sonco Ltd, 으 Batley, UK) which converted light emission $\underset{x}{0}$ into millivolt readings. Platelets $\left(75 \times 10^{6}\right)$ in $500 \mu \mathrm{l} \mathrm{Hank's} \mathrm{solution} \mathrm{were} \mathrm{tested} \mathrm{in} \mathrm{the} \mathrm{pres-}$ ence of luminol $418 \mu \mathrm{M}$ and horseradish per-응 oxidase $(720 \mathrm{mU})$. Results were expressed in terms of the change in the light emission by dividing the maximum value of light emission $\frac{7}{0}$ obtained immediately after the addition of aspirin to the platelets by the light emission ob- $\sigma$ tained with platelets in the presence of both $N$ luminol and horseradish peroxidase (basal N value). To investigate the effects of aspiring and PAF on chemiluminescence in more detailo different doses of aspirin $(20 \mu \mathrm{l}, 40 \mu \mathrm{l}, 60 \mu \mathrm{l}$, $80 \mu \mathrm{l}$, and $100 \mu \mathrm{l})$ and PAF $(25 \mu \mathrm{l}, 50 \mu \mathrm{l}, 100 \mu \mathrm{l}, \stackrel{\text { P }}{?}$ and $150 \mu \mathrm{l}$ ) were used.

GLUTATHIONE PEROXIDASE (GSH-Px) ASSAY GSH-Px activity was determined according to a method previously described. ${ }^{21}$ Washed $\delta$ platelets were resuspended in $\mathrm{PO}_{4} \mathrm{Na}$ buffer, $\mathrm{pH} 7 \cdot 4$, containing $2 \times 10^{-4} \mathrm{M}$ NADP, $10^{-2} \mathrm{M}$ ô aminocaproic acid, $10^{-2} \mathrm{M}$ ethylene diaminetetraacetic disodium salt, and $10^{-3} \mathrm{M}$ 2 -mercaptoethanol. The suspension was frozen in liquid nitrogen and the supernatant was obtained by centrifugation $(30000 \mathrm{~g}$ for 10 minutes). Five $\mu l$ of the platelet lysate were taken and redissolved in $100 \mu \mathrm{l}$ Tris- $\mathrm{HCl} 1 \mathrm{M}$ at pH 8, $20 \mu \mathrm{l} \mathrm{GHS}$ (glutathione) $0 \cdot 1 \mathrm{M}, 100 \mu \mathrm{l}$ GSH-R (glutathione reductase) $10 \mathrm{U} / \mathrm{ml}$, 


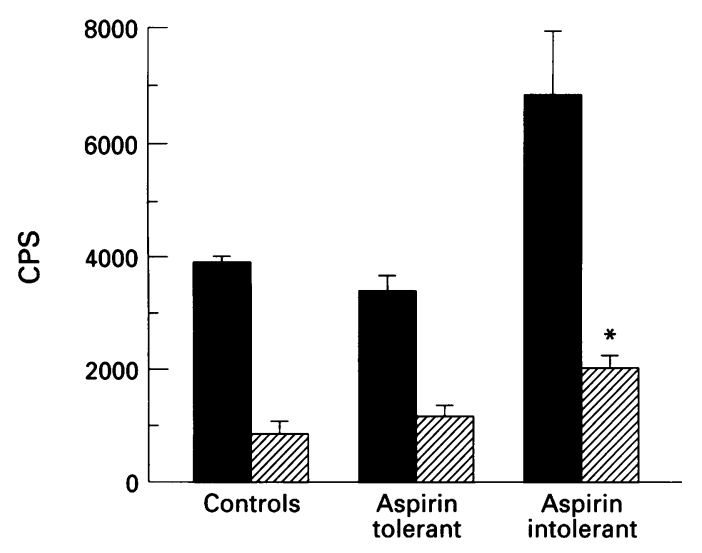

Figure 2 Arachidonic acid metabolite basal values: $\square=$ cyclo-oxygenase products (TXB,$P G F_{2 x} P G E_{2}$, and $H H T)$ and $\mathbb{Z}=$ lipoxygenase products (12-HETE and metabolite X). Aspirin-intolerant patients had a higher metabolic activity than the other two groups, although the difference was only statistically significant for lipoxygenase products $(* p<0.005)$. CPS = counts per second.

$100 \mu \mathrm{l}$ NADPH $2 \mu \mathrm{M}$, and $665 \mu \mathrm{l}_{2} \mathrm{O}$. The mixture was incubated at $37^{\circ} \mathrm{C}$ for 10 minutes. The reaction was initiated by adding $10 \mu \mathrm{l} t$ butyl-hydroperoxide $7 \mu \mathrm{M}$ (1/1000). Conversion of NADPH to NADP was made by measuring the change of absorbance at $340 \mathrm{~nm}$ using a spectrophotometer (Kontron, Uvikon 860). The non-enzymatic oxidation of NADPH was determined using an identical assay system without the addition of the platelet lysate (control tube). The increment of absorbance in the control tube was subtracted from that obtained with the platelet lysate to determine the true GSH-Px activity. Values obtained were expressed as $\mathrm{mU}$ per $10^{9}$ platelets.

\section{DATA ANALYSIS}

Analysis of variance and Kruskal-Wallis tests were used to evaluate differences among the three groups. The Mann-Whitney test was used to assess differences between the two groups and the $\chi^{2}$ test and Pearson's correlation were also used when appropriate. Significance level in all tests was $\mathrm{p}<0 \cdot 05$.

\section{Results}

ARACHIDONIC ACID METABOLITES

Basal values

Platelets from aspirin-intolerant patients showed a significant basal activity and produced more cyclo-oxygenase and lipoxygenase products than platelets from both aspirin-tolerant patients and healthy subjects. No differences were observed between aspirin-tolerant patients and healthy subjects. In order to analyse the two pathways of platelet arachidonic acid metabolism, cyclo-oxygenase products $\left(\mathrm{TXB}_{2}, \mathrm{PGF}_{2 x}, \mathrm{PGE}_{2}\right.$, and HHT) were grouped and compared with lipoxygenase metabolites (12-HETE and metabolite X). Aspirinintolerant asthmatics showed more cyclooxygenase and lipoxygenase products than the other groups (fig 2). Although the two metabolic pathways were more active in aspirin-intolerant patients, only the increase in lipoxygenase products reached statistical significance between the aspirin-intolerant and the control group ( $p=0.005)$.

\section{Response to PAF}

The response of cyclo-oxygenase and lipoxygenase metabolites to PAF is shown in fig 3. 12-HETE was the metabolite that increased most in the three groups. No significant changes were detected for $\mathrm{PGE}_{2}$ and $\mathrm{PGF}_{2 x}$ PAF caused a significant increase in $\mathrm{TXB}_{2}$ and HHT release only in aspirin-intolerant patients. It stimulated the lipoxygenase pathway more (12-HETE increased $255 \%$ for the three groups
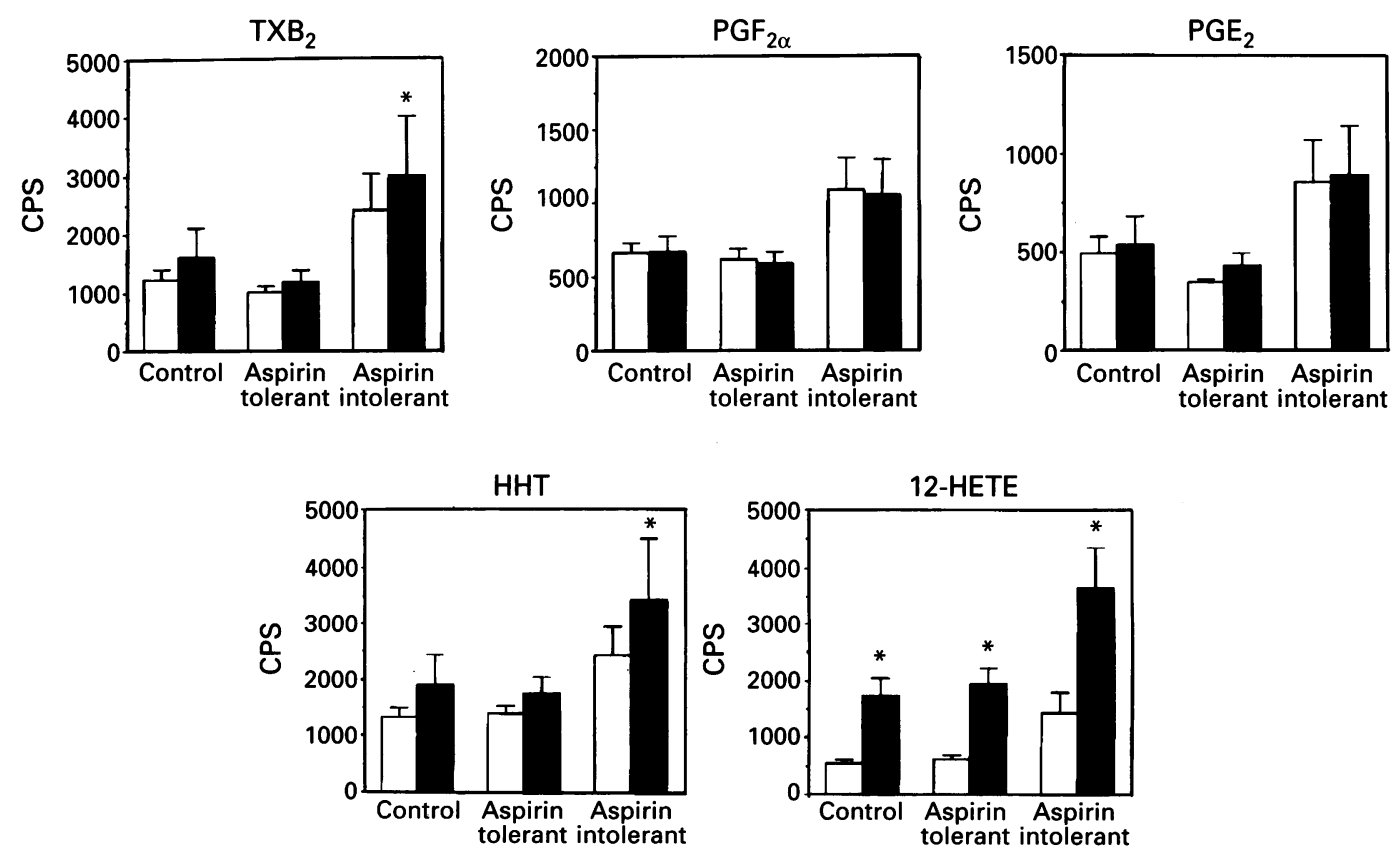

Figure 3 Platelet arachidonic acid metabolite response to platelet activating factor (PAF). 12-HETE was the metabolite that increased most in the three groups. No significant changes were detected for $P G E_{2}$ and $P G F_{2 x}$. PAF caused a significant increase in $\mathrm{TXB}_{2}$ and HHT release only in aspirin-intolerant patients $(* p<0 \cdot 05)$. CPS $=$ counts per second. 

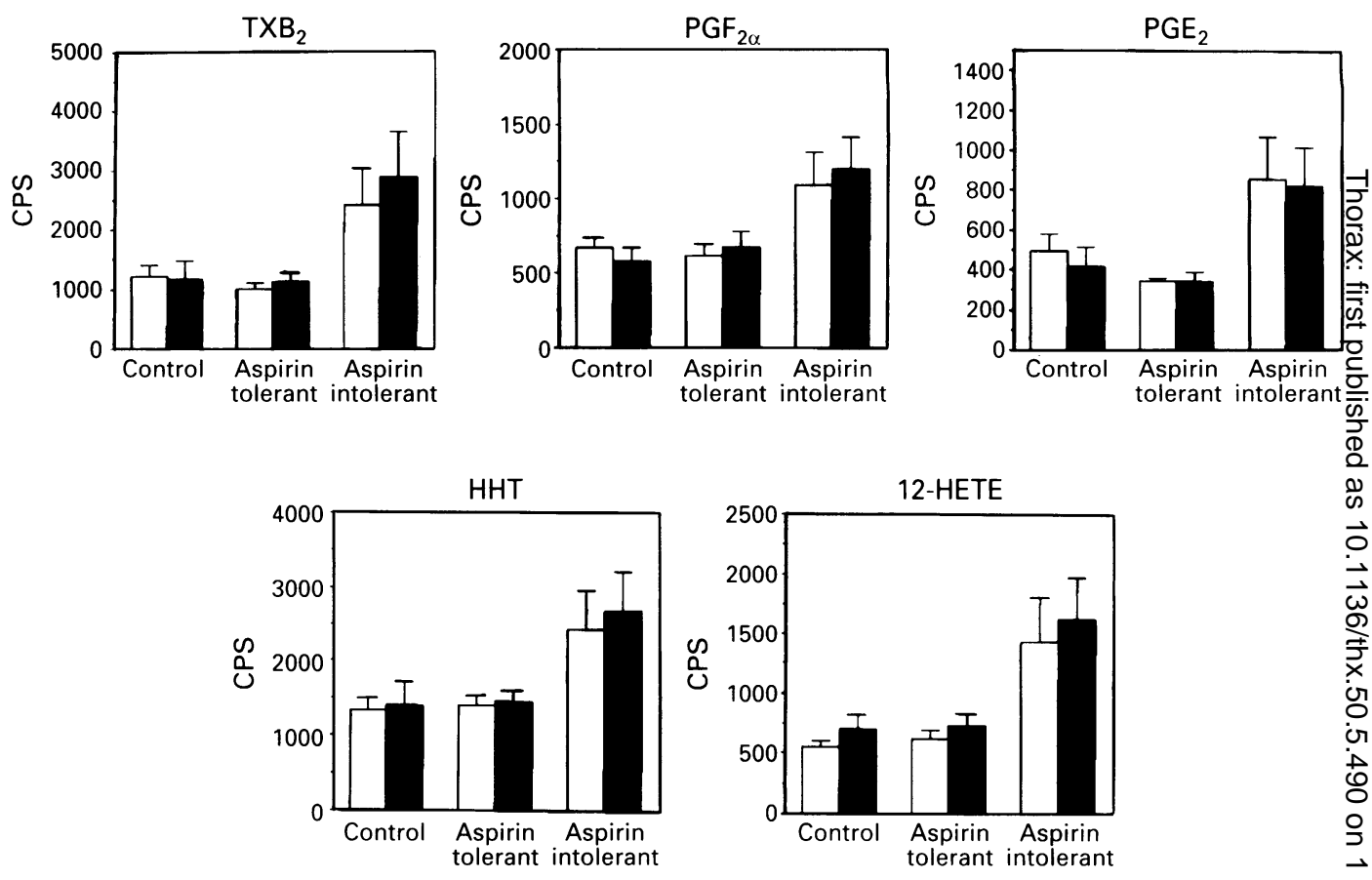

Figure 4 Platelet arachidonic acid metabolite response to aspirin. No differences in the production of either cyclooxygenase or lipoxygenase metabolites were detected among the three groups after incubation with aspirin. CPS $=$ count per second.

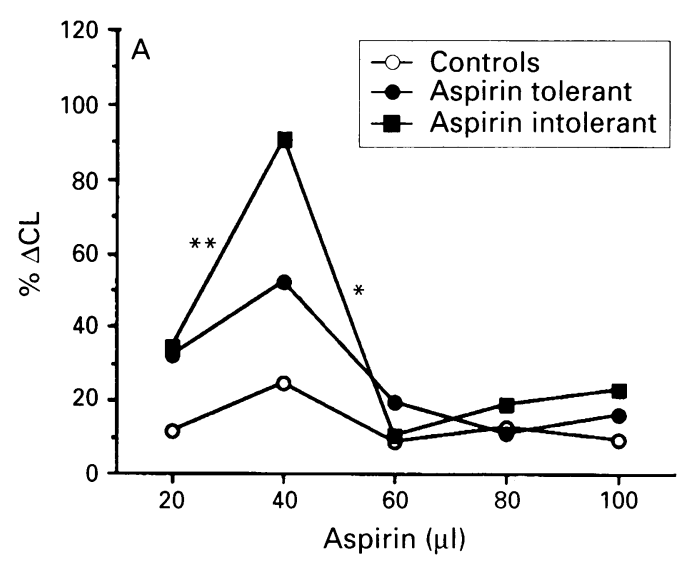

taken together) than the cyclo-oxygenase pathway (a $20 \%$ increase in $\mathrm{TXB}_{2}, \mathrm{PGE}_{2}, \mathrm{PGF}_{2 x}$, and HHT production for the three groups) $(\mathrm{p}<0 \cdot 0001)$.

Response to aspirin

Aspirin caused the least quantitative changes and none of these changes reached statistical significance (fig 4). No differences in the production of lipoxygenase metabolites were de- $\frac{0}{3}$ tected among the three groups after incubation with aspirin.

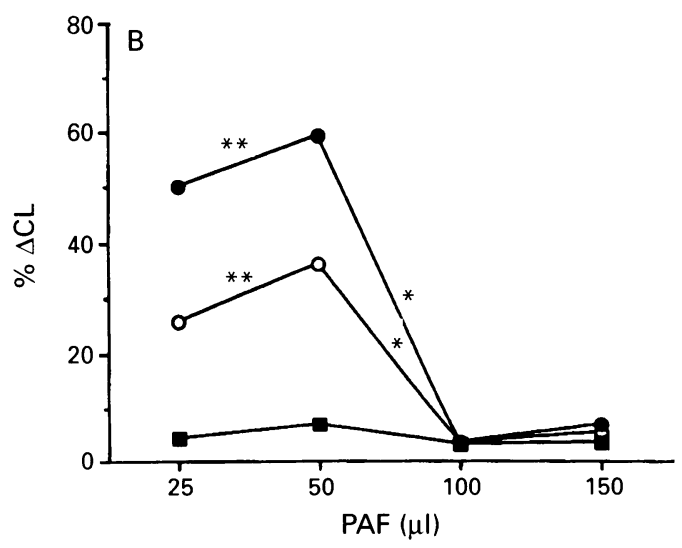

Figure 5 Production of oxygen-free radicals measured by chemiluminescence $(C L)$ with different doses of $(A)$ aspirin and $(B)$ platelet activating factor (PAF). Results are expressed in terms of change (\%CL) in light emission by dividing the maximum value of $C L$ obtained immediately after the addition of aspirin to platelets, by the CL obtained with platelets in the presence of both luminol and horseradish peroxidase (basal value). (A) The maximal burst of $C L$ was obtained with $40 \mu l$ aspirin. Changes were statistically significant only for asthmatics (Friedman two way ANOVA and Wilcoxon's tests: $\left.{ }^{*} p<0 \cdot 01,{ }^{* *} p<0 \cdot 001\right)$. Although a greater response was observed in aspirin-intolerant patients than in both aspirin-tolerant and healthy subjects, differences did not reach significance. (B) The maximal burst of $C L$ was obtained with $50 \mu l$ PAF in aspirintolerant patients. In platelets from aspirin-intolerant patients no increase in light emission could be detected. Changes were significant for aspirin-tolerant patients and healthy subjects (Friedman two way ANOVA and Wilcoxon's tests, ${ }^{*} p<0 \cdot 01,{ }^{* *} p<0 \cdot 001$ ).

\section{PLATELET CHEMILUMINESCENCE}

For technical reasons only nine patients from each group were studied. PAF and aspirin- $\widetilde{N}$ induced platelet chemiluminescence at differ- $N$ ent doses is presented in fig 5. Platelets from $\mathrm{N}$ aspirin-sensitive patients showed the maximal 0 burst of chemiluminescence after being in- 0 cubated with $40 \mu \mathrm{l}$ aspirin. The addition of $\frac{\bigodot}{\mathbb{Q}}$ different doses of aspirin to platelets from $\stackrel{\infty}{+}$ healthy and aspirin-tolerant asthmatic subjects $\frac{T}{0}$ produced a mild and non-significant increase $\frac{O}{\mathbb{D}}$ in light emission whereas in aspirin-intolerant $\frac{?}{\mathbb{D}}$ asthmatics a moderate and significant response $\stackrel{\varnothing}{\circ}$ in light emission was detected with $40 \mu \mathrm{l}$ ? aspirin. Although aspirin-intolerant patients 8 showed higher chemiluminescence values than the other two groups, the difference was not significant. Only three of the nine aspirin-? intolerant patients, however, showed a marked increase in chemiluminescence.

PAF produced a considerable increase in light emission in aspirin-tolerant patients and healthy subjects. However, in contrast to aspirin, PAF had no effect in aspirin-intolerant patients. In aspirin-tolerant patients $25 \mu$ l and $50 \mu \mathrm{l}$ PAF significantly increased chemi- 


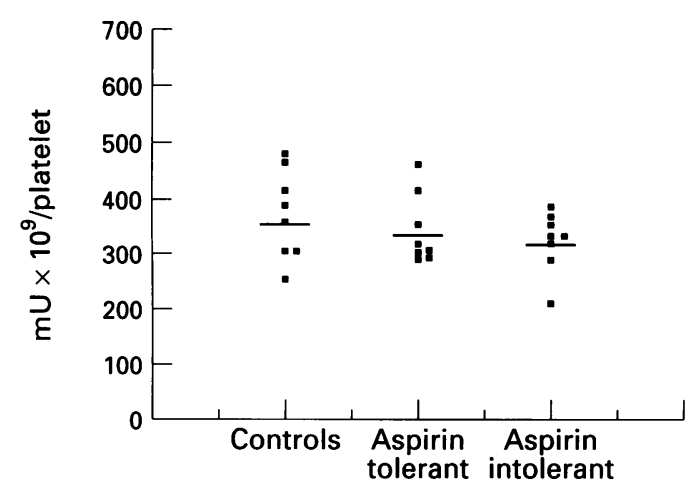

Figure 6 Glutathione peroxidase activity for the three groups. Values are expressed as $m U \times 10^{9} /$ platelet. No differences were found among the three groups (KruskalWallis test).

luminescence $(p<0.005)$. The light emission response was also statistically significant with $50 \mu \mathrm{l}$ PAF in healthy subjects $(\mathrm{p}<0.005)$. In aspirin-intolerant patients the increase in light emission after incubation with $40 \mu$ laspirin was significantly higher than after incubation with PAF $(50 \mu \mathrm{l})(\mathrm{p}<0.05)$.

\section{GSH-Px ACTIVITY}

For technical reasons only eight subjects from each group were studied. The mean (SD) results of the GSH-Px assay were 369.6 (82) $\mathrm{mU}$ $\times 10^{9} /$ platelet in controls (group 1); 340.6 (64) $\mathrm{mU} \times 10^{9} /$ platelet in aspirin-tolerant asthmatics (group 2); and 330.8 (58) $\mathrm{mU} \times 10^{9} /$ platelet in aspirin intolerant asthmatics (group 3) (fig 6). No significant differences in GSHPx determinations were found either between healthy and asthmatic subjects, or between both groups of patients.

\section{Discussion}

There were three major findings in this study. Firstly, platelet arachidonic acid metabolism was more active in aspirin-intolerant patients than in both aspirin-tolerant and healthy subjects. Secondly, no differences in the response of arachidonic acid metabolism to either aspirin or PAF were found among the three groups and, finally, incubation with aspirin but not with PAF caused an increase in light emission in aspirin-intolerant patients whereas in aspirintolerant patients PAF rather than aspirin was the more potent stimulus for oxygen-free radical production.

The overproduction of arachidonic acid metabolites detected in our study could result from an actual increase in the release of these products, but may also be the consequence of differences in the kinetics of the release of these metabolites between aspirin-intolerant patients and aspirin-tolerant and healthy subjects. The method used in this study (single time point) does not allow this question to be answered. Only a kinetic analysis with different substrate concentrations would allow us to elucidate the cause of the overactivity of the arachidonic acid pathway in aspirin-intolerant patients.

The mechanism responsible for the different response in light emission between aspirinintolerant and aspirin-tolerant patients to as- pirin and PAF is also unclear, but our findings suggest the presence of two binding sites for PAF - a phenomenon recently described in eosinophils. ${ }^{223}$ In these cells occupation of the high affinity PAF receptor correlates with prostanoid release whereas occupancy of the low PAF receptor is associated solely with the generation of ORL. In our study we did not defect differences in prostanoid release after PAF stimulation among the three groups; the same stimuli, however, increased the production of oxygen-free radicals in aspirin-tolerant patients and healthy subjects but not in aspirin-intolerant asthmatic patients. This phenomenon suggests the possible existence in platelets, as in eosinophils, of two PAF receptors and that in aspirin-intolerant asthmatic subjects the low affinity PAF receptor is downregulated for unknown reasons. Our results reinforce previous findings which suggest that the production of oxygen-free radicals may differentiate aspirin-tolerant from aspirin-intolerant asthmatics. Although Ameisen et al ${ }^{9}$ have suggested that the increased oxygen-free radical production by aspirin in aspirinintolerant patients is caused by the generation of lipoxygenase metabolites, our results do not support this hypothesis. Similarly, Nizankowska et $a l^{24}$ could not detect differences in the production of 12-HETE between healthy subjects and NSAID-intolerant patients after aspirin provocation.

The second hypothesis suggests that the supposed specific abnormality of arachidonic acid metabolism is localised in the cyclo-oxygenase pathway. Exogenous arachidonic acid is rapidly transformed by platelets into the prostaglandin endoperoxide $\mathrm{PGG}_{2}$. This metabolic step is catalysed by cyclo-oxygenase. $\mathrm{PGG}_{2}$ is a very short lived compound which is rapidly transformed into $\mathrm{PGH}_{2}$ via a hydroperoxidase. ${ }^{25}$ Ameisen et $a l^{926}$ have shown, in a series of experiments, that alterations in the synthesis of $\mathrm{PGH}_{2}$ endoperoxides may be responsible for the generation of oxygen-free radicals by the platelets of patients with NSAID intolerance. These authors have demonstrated that two different antagonists of the platelet $\mathrm{PGH}_{2}$ receptor (trimetoquinol and $\mathrm{PTAOH}$ ) may precipitate aspirin-like reactions in platelets from aspirin-intolerant asthmatics, and that this reaction may be prevented by the addition of non-metabolisable analogues of the unstable $\mathrm{PGH}_{2}$. These results suggest that aspirin-induced asthma might not be merely the consequence of a cyclo-oxygenase blockade, as previously proposed, and that an ongoing synthesis of $\mathrm{PGH}_{2}$, and the interaction of $\mathrm{PGH}_{2}$ with its platelet receptor, might play a part in aspirin-induced asthma. ${ }^{10}$

We only detected an increased production of oxygen-free radicals in $33 \%$ of our aspirinintolerant patients, a figure which contrasts with the study of Ameisen $e t a l^{9}$ who found a $100 \%$ positive aspirin-specific increase in the generation of oxygen-free radicals after stimulation of platelets from aspirin-sensitive patients. Other authors, however, have also failed to demonstrate this universal response of aspirin-sensitive patients. ${ }^{12132627}$ In keeping 
with our results, Pearson and Suarez-Mendez ${ }^{12}$ reported that only $32 \%$ of their aspirin-sensitive asthmatics released oxygen-free radicals in the presence of aspirin. Other authors, however, could not detect an abnormal aspirin-induced platelet release of oxygen metabolites either by chemiluminescence or other methods. ${ }^{1327}$ Differences in the methodology and in the patients studied might account for these discrepancies.

In keeping with Stone et $a l^{28}$ we were unable to detect differences in platelet GSH-Px activity between asthmatic and healthy subjects. These findings contrast with those of Hasselmark et $a l^{13}$ who found significantly lower GSH-Px activity in patients with intrinsic asthma than in a control group. Pearson and Suarez-Mendez ${ }^{12}$ also found that the mean platelet GSH-Px activity was significantly lower in subjects with aspirin-induced asthma than in a healthy control group. Nevertheless, this difference could not be detected between aspirin-tolerant asthmatics and the control group. Clinical differences may account for the discrepancy in these results.

A more active disease is probably associated with an increased production of reactive oxygen radicals which may inactivate the GSH-Px. This hypothesis is supported by the observation of Bibi et $a l^{29}$ who demonstrated a close correlation between asthma severity and erythrocyte GSH-Px activity. They found that GSHPx levels were significantly lower during exacerbation of asthma than during stable clinical conditions. Similarly, Pearson and SuarezMendez $^{12}$ also observed that platelet GSH-Px activity was lower in patients with symptoms of severe asthma than in those with mild disease. We think that the platelet GSH-Px activity of our patients and healthy subjects did not differ because only mild asthmatics were included in the study. Moreover, asthmatics studied by Malmgren et al $^{11}$ were not receiving any kind of treatment, while our patients and those in the study by Stone $e t a l^{28}$ were treated with inhaled steroids (some of the latter were also receiving oral steroids). The possible effects of these treatments on platelet levels of GSH-Px cannot be overlooked.

In summary, our study shows a different in vitro response to aspirin and PAF of platelets from aspirin-tolerant and aspirin-intolerant asthmatic subjects. These results support previous findings suggesting that the production of oxygen-free radicals by platelets may differentiate aspirin-tolerant from aspirin-intolerant patients. Although the mechanisms involved in this response are unclear, our study does not support the presumed increase in lipoxygenase metabolites by inhibition of the cyclo-oxygenase pathway as the cause of oxygen-free radical production. Reduced platelet GSH-Px activity does not seem to be responsible for the pathogenesis of this intriguing phenomenon.

Supported by grants from Comisión Asesora Interministerial Ciencia y Tecnologia (CAICYT) (PB 86/0594) and Fondo Investigaciones Sanitarias (FIS) $(89 / 0386)$
1 Szczeklik A. Analgesics, allergy and asthma. Drugs 1986;32 (Suppl 4):148-63.

2 Szczeklik A, Gryglewski RJ, Czerniawska-Mysik G. Clinical patterns of hypersensitivity to non-steroidal antiinflammatory drugs and their pathogenesis. $\mathcal{f}$ Allergy Clin Immunol 1973;60:276-84.

3 Stevenson DD, Lewis RA. Proposed mechanisms of aspirin sensitive reactions. F Allergy Clin Immunol 1987;80:788-90.

4 Bianco S, Robushi M, Petrigni G. Aspirin sensitivity in asthmatics. BMf 1981;282:146.

5 Szczeklik A, Gryglewsky RJ, Czerenawka-Mysik B. Relationship of inhibition of prostaglandin biosynthesis analgesics to asthma attacks in aspirin-sensitive patients. $B M F 1975 ; 1: 67-9$.

6 Szczeklik A. Aspirin-induced asthma as a viral disease. Clin Allergy 1988;18:15-20.

7 Christie PE, Tagari P, Ford-Hutchinson AW, Charlesson, $\frac{\omega}{}$ Chee P, Arm JP, Lee TH. Urinary $\mathrm{LTD}_{4}$ concentration increase after aspirin challenge in aspirin-sensitive asthmatic subjects. Am Rev Respir Dis 1991;143:1025-9.

8 Picado C, Ramis I, Roselló J, Prat J, Bulbena O, Plaza V, et al. Release of peptide leukotrine into nasal secretions after local instillation of aspirin in aspirin-sensitive asthmatic patients. Am Rev Respir Dis 1992;145:65-9.

9 Ameisen JC, Capron A, Joseph M, Maclouf J, Vorng H, Pancre V, et al. Aspirin-sensitive asthma: abnormal platelet response to drugs inducing asthmatic attacks. Int Archs $\vec{x}$ Allergy Appl Immunol 1985;78:438-48.

10 Ameisen JC, Capron A. Aspirin-sensitive asthma. Clin Exp Allergy 1990;20:127-9.

11 Malmgren R, Unge G, Zetterstrom O, Theorell H, de Wahl $\mathrm{K}$. Lowered glutathione-peroxidase activity in asthmatic patients with food and aspirin intolerance. Allergy 1986; $ᄋ$ 41:43-5.

12 Pearson J, Suarez-Mendez J. Abnormal platelet hydrogen peroxide metabolism in aspirin hypersensitivity. Clin $\operatorname{Exp}>$ Allergy 1990;20:157-63.

13 Hasselmark L, Malmgren $R$, Unge $G$, Zetterström $O$. Lowered platelet glutathione peroxidase activity in patients $\vec{\theta}$ with intrinsic asthma. Allergy 1990;45:523-5.

14 Nicholls P. Contributions of catalase and glutathione peroxidase to red cell peroxide removal. Biochim Biophys Acta 1972;279:306-9.

15 Flohé L, Zimmerman R. GSH-induced high-amplitude swelling of mitrochondria. In: Flohé L, Benohr HC, Sies $\mathrm{H}$, Waller HD, Wendel A, eds. Glutathione. Stuttgart: H, Waller HD, Wendel A, eds. Glutathione. Stutgart:

16 Levine RF, Fedorko ME. Isolation of megakaryocytes from guidea pig femoral marrow. Successful harvest made pos- $\overline{\bar{O}}$ sible with inhibitors of platelet aggregation: enrichment $\mathrm{O}$ achieved with a two-step separation technique. $\mathcal{f}$ Cell Biol 3 1976;69:159-72.

17 Rao GRH, Escolar G, White JG. Epinephrine preserves the inhibitory influence of aspirin on platelet-vessel wall interactions. Thromb Res 1986;44:65-74.

18 Born GVR, Cross MJ. The aggregation of blood platelets. 음 f Physiol 1963;168:178-95.

19 Rittenhouse-Simmons S. Release and metabolism of ar- $\underset{x}{ }$ achidonate in human platelets. In: Gordon JI, ed. Platelets in biology and pathology. Vol. 2. Amsterdam: Elsevier-North Holland Biomedical Press, 1981:349-72.

20 Yen SS, Morris HG. An imbalance of arachidonic acid $\mathrm{O}$ metabolism in asthma. Biochem Biophys Res Commun 1981; 2:774-9.

21 Vives JL, Aguilar JLI. Manual de técnicas de laboratorio en hematologia. Barcelona: Salvat SA, 1988:324-5.

22 Kroegel C, Yukawa T, Westwick J, Barnes PJ. Evidence for two platelet activating factor receptors on eosinophils: dissociation between PAF-induced intracellular calcium $N$ mobilization degranulation and superoxides anion genmobilization degranulation and superoxides anion gen-
eration in eosinophils. Biochem Biophys Res Commun 1989; $N$
$1.511-21$ 1:511-21.

23 Kroegel C, Warner JA, Giembycz MA, Matthys H, Lich- W tenstein LM, Barnes PJ. Dual transmembrane signaling 0 mechanisms in eosinophils: evidence for two functionally distinct receptors for platelet activating factor. Arch Allergy Appl Immunol 1992;99:226-9.

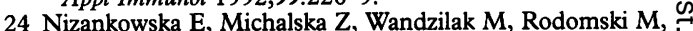
Marcinkiewicz E, Gryglewski RJ, et al. An abnormality of $\tau$ arachidonic acid metabolism is not a generalized phenomenon in patients with aspirin-induced asthma. Eicosanoids 1988;1:45-8.

25 Lagarde $M$. Metabolism of fatty acids by platelets and the functions of various metabolites in mediating platelet function. Prog Lipid Res 1988;27:135-52. PV PV, Tonnel AB, et al. Aspirin-sensitive asthma. Involvement of the prostaglandin endoperoxidase receptor in the abnormal platelet response to drugs inducing asthmatic attacks. Am Rev Respir Dis 1987;135 (Suppl):A311.

27 Schmitz-Schumann M, De Souza V, Menz G, Schäufele A, Von Felten A, Matthys $\mathrm{H}$, et al. Reduced production of oxygen-free radicals of platelets in aspirin-induced asthma. Agents Actions 1989;28 (Suppl): 195-203.

28 Stone J, Hinks LJ, Beasley R, Holgate ST, Clayton BA Reduced selenium status of patients with asthma. Clin Sci 1989;77:495-500

29 Bibi H, Schlesinger M, Tabachnik E, Schwartz Y, Iscovitz $\mathrm{H}$, Iaina A. Erythrocyte glutathione peroxidase activity in asthmatic children. Ann Allergy 1988;61:339-40. 